\title{
Estructura de capital, concentración de propiedad y política de dividendos como factores determinantes de problemas de agencia en empresas chilenas
}

\author{
Roberto Vallejos Villa \\ Facultad de Economía y Negocios \\ Universidad de Chile \\ rvallejos@fen.uchile.cl
}

\begin{abstract}
The objective of this paper is to analyze the capital structure, the concentration of property and the policy of dividends as determinants of the problems of agency in Chilean companies. As a measure of agency costs, the asset turnover ratio was used as a benchmark for management efficiency in the use of the company's assets, which is an inverse measure of agency costs.

The results show that a higher level of indebtedness seems to favor the discretional behavior of managers. However, there is also evidence that when the debt is a short-term debt, the divergence of interest decreases between shareholders and managers.

The concentration of property on the other hand has a nonlinear effect on the costs of agency, which means that, first, it acts as a mechanism to attenuate managerial discretion, but when it reaches a certain level, an effect of expropriation of major shareholders over minor shareholders seems to be produced. In relation to the dividend policy, a positive relationship is observed with efficiency in the use of assets, mainly in the presence of high growth opportunities.
\end{abstract}

Keywords: Capital structure; Property concentration; Dividend policy; Agency problems 


\begin{abstract}
Resumen
El objetivo de este trabajo es analizar la estructura de capital, la concentración de propiedad y la política de dividendos como factores determinantes de los problemas de agencia en las empresas chilenas. Como medida de costos de agencia se utilizó el ratio de rotación de activos como referencia de la eficiencia directiva en el aprovechamiento de los activos de la empresa, lo que constituye una medida inversa de costos de agencia.

Los resultados muestran que un mayor nivel de endeudamiento pareciera favorecer el comportamiento discrecional por parte de los directivos. Sin embargo, también hay evidencia de que cuando la deuda es a corto plazo la divergencia de intereses disminuye entre accionistas y directivos. La concentración de propiedad por su parte arroja un efecto no lineal sobre los costos de agencia, es decir, en primera instancia actúa como un mecanismo para atenuar la discrecionalidad directiva pero al llegar a un cierto nivel, pareciera producirse un efecto de expropiación de los accionistas mayoritarios sobre los minoritarios. En relación a la política de dividendos, se observa una relación positiva con la eficiencia en la utilización de los activos principalmente en presencia de altas oportunidades de crecimiento.
\end{abstract}

Palabaras clave: Estructura de capital; concentración de propiedad; política de dividendos; problemas de agencia

\title{
1. Introducción
}

La literatura financiera ha estudiado a lo largo del tiempo las implicancias de los problemas de agencia dentro de la empresa, de hecho, podemos encontrar un gran número de investigaciones que han estudiado la relación entre los mecanismos de gobierno corporativo y el valor de la firma. No obstante, mientras dichos estudios proveen resultados útiles para comprender la implicancia y consecuencias que tienen los problemas de agencia entre directivos y accionistas, los factores determinantes de los costos de agencia han tenido escasa atención en la literatura. 
Entre aquellos autores que efectivamente han abordado la medición de los costos de agencia y examinado sus determinantes, se encuentran importantes trabajos, como Ang et al. (2000) quienes encuentran que los costos de agencia son mayores cuando la empresa es manejada por directivos externos, y que disminuyen a medida que se incrementan los accionistas internos y existe un monitoreo externo bancario. Singh y Davidson (2003) encuentran que un mayor nivel de accionistas internos ${ }^{1}$ llevará a una convergencia de intereses entre directivos y accionistas externos ${ }^{2}$, sin embargo, contrariamente a la teoría, encuentran que el endeudamiento en grandes empresas está positivamente relacionado con costos de agencia. Florackis (2008) muestra que las oportunidades de crecimiento son un determinante significativo del nivel de costos de agencia de la empresa y que la deuda a corto plazo es un mecanismo para reducir problemas de agencia.

Sin embargo, la mayor parte de los trabajos previos son realizados para economías desarrolladas como EE.UU., UK y Australia, las que poseen características del sistema financiero muy distintas a las que se observan en economías emergentes. Por lo que resultaría interesante analizar cómo algunos mecanismos de gobierno corporativo impactan en los costos de agencia en un sistema financiero y entorno institucional propio de una economía emergente como la chilena. Es por esto que en el presente trabajo se busca analizar algunos mecanismos de control interno como la estructura de capital, la concentración de propiedad y la política de dividendos en su papel de aminorar los costos de agencia surgidos de la separación de propiedad y control.

Bajo esa perspectiva, el objetivo de este trabajo es analizar la estructura de capital, la concentración de propiedad y la política de dividendos como factores determinantes de los problemas de agencia en las empresas chilenas.

\footnotetext{
${ }^{1}$ Aquellos accionistas que participan en la toma de decisiones.

${ }^{2}$ Accionistas minoritarios.
} 
Al igual que Ang et al., (2000) y Singh y Davidson (2003), en este trabajo se utiliza el ratio de rotación de activos (ventas totales sobre activos totales) como una medida inversa de los costos de agencia. Este ratio puede ser interpretado como una medida de la eficiencia en la utilización de activos y la habilidad que tienen los directivos para manejarlos de manera óptima y productiva. Así, los directivos de las empresas con bajos índices de rotación de activos, son más propensos a tomar decisiones subóptimas de inversión, hacer un esfuerzo insuficiente y utilizar los recursos de la empresa para la adquisición de activos improductivos.

De esta manera, el presente trabajo realiza dos contribuciones. Primero, se evalúa directamente el impacto de los mecanismos de control corporativos sobre la medida de costo de agencia en vez de hacerlo en el valor o rendimiento de la firma. Y en segundo lugar, aporta evidencia empírica acerca de la discrecionalidad directiva en un entorno institucional distinto al de los países anglosajones, que son los comúnmente estudiados en la literatura financiera.

Para alcanzar el objetivo propuesto el presente trabajo se estructura en cinco partes. En la segunda sección se realiza una revisión del marco teórico a base de los mecanismos de control que se estudiarán en donde además se establecerán las respectivas hipótesis a analizar. En la tercera parte se presentan los datos y las variables a estudiar, así como también la descripción de la metodología estadística a desarrollar. En el cuarto apartado se expondrán los resultados de los análisis descriptivos y multivariantes realizados. Y finalmente en la quinta parte se presentan las principales conclusiones. 


\section{Marco teórico e hipótesis}

\section{A. Costo de agencia}

La literatura financiera ha estudiado a lo largo del tiempo las implicancias de los problemas de agencia dentro de la empresa. La teoría de agencia estudia los contratos en los que una persona (el principal) contrata a otra persona (el agente) para realizar un determinado servicio en su favor en donde se le otorga una cierta autoridad para tomar decisiones en la empresa. Entonces, si ambas partes son maximizadoras de utilidades se infiere que el agente no actuará siempre en los mejores intereses del principal. Además, debido a la simetría de información en donde el agente comúnmente posee mejor ingformación que el principal en cuanto a hechos relevantes, el principal no puede asegurarse por su cuenta que el agente está actuando acorde a lo prometido y acordado en el contrato. Por tanto, el agente tiene un incentivo para actuar de forma oportunista, escatimando en la calidad de su actuación, o incluso desviando a sí mismo algo que le correposne al principal.

Jensen y Meckling (1976) definen los costos de agencia como la suma de tres elementos, los que son los gastos de monitoreo de parte del principal, los gastos en compromiso efectuados por el agente (bonding costs) y el costo residual.

El gasto de monitoreo en que incurre el principal corresponde al costo de las medidas de supervisión para establecer y controlar el cumplimiento del contrato ante el previsible oportunismo por parte del agente. Entre estos costos se incluyen la realización del contrato, incentivos al agente, premios a los riesgos asumidos, supervisión y control de las actividades relaizadas por los directivos.

Los gastos en compromisos efectuados por el agente (bonding costs) corresponde a los costos en los que incurre el agente surgidos por la relación con el principal. Entre estos gastos están los costos de su promesa de no actuar en contra del interés del principal, 
los costos de autocontrol, o costos de garantía consistentes en la obligación de indemnizar por posibles daños al principal.

Finalmente, a causa de que es imposible asegurar que las acciones del agente estarán totalmente alineadas con los intereses del principal aun cuando ambas partes incurren en costos para lograrlo, siempre habrá un costo residual. Este costo residual corresponde al equivalente monetario de la reducción de bienestar que sufre el principal producto de la divergencia de intereses con el agente.

Kraakman et al. (2009) mencionan tres problemas de agencia generales bajo la perspectiva de la relación principal-agente. El primero abarca el conflicto entre los propietarios de la firma y los directivos contratados. El problema está en asegurar que los directivos (agentes) actúen acorde con los intereses de los dueños (principales) en lugar de seguir los suyos. El segundo problema de agencia radica en el conflicto entre los accionistas mayoritarios y los minoritarios. En este caso, los accionistas minoritarios pueden ser considerados como los principales, mientras que los accionistas mayoritarios serían los agentes. Por ello, el problema en este caso está en asegurar que los accionistas mayoritarios actúen en función de todos los accionistas, y no solo en favor de ellos mismos. El tercer problema de agencia nace del conflicto entre la empresa misma $^{3}$ y las otras partes con quien la empresa mantiene contratos, como acreedores, empleados y clientes. Aquí el problema surge en asegurar que la empresa, como agente, no caiga en un comportamiento oportunista hacia los distintos principales mencionados anteriormente. En este caso, la empresa puede incurrir en acciones como la expropiación a acreedores, explotación a empleados o engaños a los clientes.

\footnotetext{
${ }^{3}$ Incluyendo en forma particular a sus propietarios.
} 


\section{B. Estructura de capital}

Como se ha señalado con anterioridad, no siempre los directivos toman las decisiones que llevan a una política de inversiones óptima, produciendo conflictos de intereses entre directivos y accionistas (Fama y Jensen, 1983), entre accionistas y acreedores (Smith y Warner, 1979) y conflicto entre accionistas mayoritarios y minoritarios (La Porta et al., 1999).

Cuando en una empresa nos encontramos con divergencias de intereses entre directivos, accionistas o bonistas, usualmente lo relacionamos a problemas de asimetría de información y flujos de caja libre (Jensen, 1986). Una forma de reducir estos problemas es mediante el endeudamiento, en donde una deuda bancaria por ejemplo entregará señales positivas al mercado respecto de la solvencia del prestatario, y por tanto, mitigará la información asimétrica entre los managers y accionistas (Jensen, 1986; Stulz, 1990).

Concretamente la estructura de capital es utilizada como mecanismo de control directivo. De esta manera, el endeudamiento disminuye el consumo de beneficios no pecuniarios en desmedro de la riqueza de los dueños, ya que una empresa más apalancada se tornará más riesgosa, lo que constituye una amenaza en la estabilidad del empleo de los directivos provocando que estos aminoren ese tipo de gastos. Por tanto, la utilización de deuda funciona como mecanismo de disciplina directiva, por lo que se debería dar una relación inversa entre los costos de agencia y el endeudamiento de la empresa (López y Saona 2007).

Por otra parte, el plazo de la deuda podría tomar relevancia para mitigar los problemas de agencia. El uso de deuda a corto plazo disminuye los costos de agencia relacionados a la subinversión (Myers, 1977) y la sustitución de activos, los que surgen de los conflictos de agencia entre los accionistas de la empresa y los acreedores. Esto se debe a que la deuda a corto plazo hace la renegociación de forma más continua (Custódio et al., 2012). 
Además, la deuda a corto plazo también proporciona a los directivos la oportunidad para señalar información privada al mercado (Diamond, 1991).

A base de lo anterior, se formulan las siguientes hipótesis:

H1: Existe una relación negativa entre el nivel de endeudamiento y los costos de agencia.

H2: Existe una relación negativa entre la deuda de corto plazo y los costos de agencia.

\section{Concentración de propiedad}

Una forma de mitigar los problemas de agencia es mediante la concentración de propiedad. Teóricamente, los accionistas podrían jugar un papel importante en monitorear el desempeño de los directivos en donde aquellos accionistas con mayores porcentajes de propiedad tienen mayores incentivos de supervisar la dirección de la empresa y lo pueden hacer de forma más efectiva (Friend y Lang, 1988; Shleifer y Vishny 1997).

En economías emergentes se suelen encontrar estructuras de gobierno corporativo débiles, con regulación deficiente y fiscalización muy limitada. La inestabilidad que se presenta en estos mercados deficientes origina el incentivo a la concentración de propiedad de la empresa, generando algún tipo de accionista controlador pudiendo ser otra empresa, una familia, un banco o el Estado, quien mantenga un rol activo en el manejo de la empresa (Lefort y González, 2008).

Al igual que los demás países latinoamericanos, Chile se caracteriza por tener una alta concentración de propiedad y numerosos grupos económicos. Estos grupos controlan varias empresas mediante estructuras piramidales o series accionarias con derechos preferentes a voto. De esta manera, la elevada 
concentración de propiedad implica que el control corporativo es ejercido solo por un grupo de accionistas (los mayoritarios), por lo que se generan conflictos de intereses entre los accionistas controladores y los accionistas minoritarios (Lefort y González, 2008). Dichos problemas se generan básicamente cuando los accionistas mayoritarios alcanzan casi la totalidad de la propiedad de la empresa y toman decisiones en desmedro de los accionistas minoritarios, esto llevará a que la firma no se diversifique, pierda liquidez o incluso la posibilidad de expropiación de recursos por parte de los mayoritarios (Florackis, 2008).

Basado en lo anterior, el hecho de que una mayor concentración de propiedad implica una reducción en los costos de agencia no queda tan claro, por lo que se plantea la posibilidad de una relación negativa o no lineal entre ambas variables. En consecuancia, se plantea la siguiente hipótesis:

H3: La relación entre los costos de agencia y la concentración de propiedad puede ser positiva, negativa o no lineal.

\section{Politica de dividendos}

Otro mecanismo corporativo para combatir los problemas de agencias tiene relación al pago de dividendos. En primera instancia, los directivos pueden utilizar el pago de dividendos como una señal confiable para transmitir información privada sobre flujos de caja y perspectivas futuras de la empresa, reduciendo asimetrías de información entre directivos e inversores externos.

Como medida de control de costos de agencia, la política de dividendos indica a los accionistas que los directivos se abstendrán de obtener beneficios privados a costa de ellos. Si el flujo de caja controlado por los directivos puede ser minimizado, será más difícil para ellos gastar el dinero en actividades que son en su propio beneficio. De esta forma, un mayor pago de dividendos implica una 
reducción del flujo de caja libre, restringiendo la sobreinversión por parte de los directivos, y por esta razón, conduciendo a un mejor uso de los recursos de la empresa (Dewenter y Warther, 1998).

Otra posible forma de mitigar problemas de agencia mediante la política de dividendos consiste en: cuando una empresa no posee suficientes recursos internos para financiar sus pagos de dividendos, se verá con la obligación de conseguir recursos externos. Por lo que el manejo directivo queda bajo supervisión externa, ya que la empresa se somete al escrutinio de los mercados de capitales en donde busca dichos recursos (Easterbrook, 1984).

En el caso particular de Chile, es importante señalar que la política de pago de dividendos está regulada por la Ley $\mathrm{N}^{\mathrm{o}} 18.046^{4}$, la que indica que las sociedades anónimas chilenas tienen la obligación de pagar al menos el 30\% de sus utilidades líquidas de cada ejercicio.

Con base en lo anterior, se establece la siguiente hipótesis:

H4: Un mayor pago de dividendos está negativamente relacionado con los costos de agencia.

\section{E. Oportunidades de crecimiento}

En función de una correcta toma de decisiones financieras, se hace necesario analizar la relación que existe entre los costos de agencia y las oportunidades de crecimiento de las firmas. De hecho, la magnitud de los costos de agencia relacionados a la subinversión, sustitución de activos y flujo de caja libre, se espera que varíe notoriamente de acuerdo con que si la firma posee altas o bajas oportunidades de crecimiento (Florackis, 2008).

En el caso de problemas de subinversión, las empresas más apalancadas pueden llegar a dejar pasar proyectos con VAN positivo

${ }^{4}$ Ley N ${ }^{\circ} 18.046$ sobre sociedades anónimas promulgada en el año 1981. 
porque los flujos de caja van a pagar principalmente los compromisos actuales y el excedente en valor actual no retribuiría el aporte inicial que los accionistas aportan para la inversión. Dicho problema se presenta principalmente en empresas con atractivas oportunidades de crecimiento (Myers, 1977). Por otro lado la sustitución de activos hace alusión a la posibilidad que un alto nivel de deuda incite a los directivos a ejercer una política de inversión subóptima, en donde optarán por proyectos mucho más riesgosos, debido a que en el caso de fallar, los grandes perjudicados serían los acreedores (Jensen y Meckling, 1976). Al igual que el caso anterior, la sustitución de activos se presenta con mayor frecuencia en empresas con altas oportunidades de crecimiento debido a la asimetría de información entre los accionistas y acreedores. Por último, en relación con los problemas de flujos de caja libre, las empresas con mayores oportunidades de crecimiento presentan menos problemas de este tipo, ya que dichos problemas son más frecuentes en empresas que tienen importantes reservas de efectivo y que tienden a llevar a cabo proyectos riesgosos de inversión (Jensen, 1986).

Por lo expuesto anteriormente, se espera que la efectividad de los mecanismos corporativos sobre los costos de agencia varíe dependiendo del nivel de las oportunidades de crecimiento que presente la empresa. De esta forma, si los problemas de agencia tienen relación con subinversión o asimetrías de información, los mecanismos para mitigar dichos problemas debieran ser más efectivos en empresas con mayores oportunidades de crecimiento (Smith y Watts, 1992). Por otro lado, si los problemas de agencia son asociados con el uso del flujo de caja libre, se espera que los mecanismos de gobierno corporativo que disminuyen dichos problemas tengan una función más relevante en empresas con bajas oportunidades de crecimiento (Jensen, 1986). 


\section{F. Entorno institucional}

Para efectos de esta investigación se hace necesario analizar el entorno institucional en el que se encuentra inmersa la economía chilena. Varios trabajos entre los que destacan los de La Porta et al. (1998) se han enfocado en caracterizar los sistemas financieros de cada país y de evaluar el progreso de los mecanismos de protección de sus inversionistas.

En general se pueden observar dos tipos de mercados, los que son definidos por la literatura como el modelo anglosajón y el modelo continental. El primero se caracteriza porque su regulación se basa en la jurisprudencia, permitiendo un cierto grado de autorregulación. Las empresas presentan una estructura de propiedad más dispersa o diluida, lo que dificulta el control y seguimiento de las decisiones empresariales. Además, este modelo mantiene un cuantioso mercado de bonos corporativos como fuente de financiamiento.

Por otro lado, el modelo continental se enmarca en una regulación basada en el derecho romano, lo que entorpece el camino a la autorregulación y regulación de conflictos (Lefort, 2003). Se caracteriza por tener una elevada concentración de propiedad y las empresas suelen pertenecer a grupos económicos o incluso estructuras piramidales. Es admisible señalar además que en el modelo continental existe una vigorosa industria bancaria, la que constituye la principal fuente de financiamiento.

El mercado chileno forma parte del modelo continental, en donde la regulación de las empresas chilenas se basa en el derecho romano con limitadas prácticas de autorregulación. Sin embargo a lo largo del tiempo Chile ha presentado un crecimiento económico y desarrollo institucional, lo que le ha permitido evolucionar a formas más híbridas de sistemas financieros, adquiriendo características de mercado propias del modelo anglosajón a base de legislación basada en la jurisprudencia. La principal fuente de financiamiento de las empresas chilenas es el endeudamiento bancario, aun cuando poco a 
poco se ha incrementado el mercado de bonos corporativos. La estructura de propiedad por su parte presenta las características más distintivas respecto del modelo anglosajón, en donde las firmas poseen elevados niveles de concentración de propiedad, tienen estructura piramidal, existe un gran número de grupos económicos y bastante presencia familiar (Gutiérrez,2008).

En cuanto al marco regulatorio del sistema financiero chileno, las principales leyes que rigen el mercado son la Ley de Mercado de Valores (Ley $\mathrm{N}^{\mathrm{o}}$ 18.045) y la Ley de Sociedades Anónimas (Ley N $\mathrm{N}^{\mathrm{1}}$ 18.046), ambas promulgadas en 1981.

La Ley $\mathrm{N}^{\circ} 18.045$ abarca temas como la información periódica a accionistas, información de hechos esenciales, sanciones penales y civiles, entre otros. Mientras que la Ley $\mathrm{N}^{\circ} 18.046$ comprende aspectos como el registro de accionistas, derecho a participar y votar en juntas de accionistas, composición del directorio, entre otros.

Posteriormente, el 2000 se aprobó la ley de OPA y gobiernos corporativos (Ley $N^{\circ} 19.705$ ). Entre los principales aportes de esta ley se encuentran: se incorpora la exigencia de la Oferta Pública de Acciones (OPA) obligatoria, informada y a prorrata para todos los accionistas, crea la figura de comités de directores, reconoce derechos políticos a los tenedores de ADR, entre otros aspectos para el progreso del gobierno corporativo en las empresas chilenas.

\section{Variables y metodología}

\section{A. $\quad$ Fuentes de información y variables}

Para el análisis empírico de problemas de agencia, en el presente estudio se utiliza una muestra de 113 empresas chilenas no financieras listadas en la Bolsa de Valores de Santiago. Para ello se elaboró un panel de datos desequilibrado con 1.118 observaciones comprendiendo un período desde 2003 a 2013, el que se trabajó con 
la metodología de datos de panel. Los datos se obtuvieron de la base Thomson One y de la Superintendencia de Valores y Seguros.

A continuación se describen las variables utilizadas en este trabajo, las que se basan plenamente en la revisión teórica examinada. En la tabla 1 se exponen las variables en forma resumida.

\section{Tabla 1}

\section{Definición de las variables utilizadas}

\begin{tabular}{|l|l|}
\hline VARIABLE & \multicolumn{1}{|c|}{ DEFINICIÓN } \\
\hline ROTACT & Ventas totales/ Activos totales \\
\hline DTAT & Deuda total/ Ativo total \\
\hline DCDT & Deuda de corto plazo/ Deuda total \\
\hline P1 & \% Propiedad poseída por el primer accionista \\
\hline P1CUAD & P1 al cuadrado \\
\hline FAM & Dummy que toma el valor de 1 si el primer propietario es familia y 0 en caso contrario. \\
\hline PAYOUT & Dividendos pagados/Patrimonio \\
\hline QTOBIN & (Capitalización bursátil + Deuda total)/ Activos totales \\
\hline TAM & Logaritmo natural de ventas totales \\
\hline
\end{tabular}

En primer lugar, como variable dependiente, se emplea el ratio de ventas totales sobre activos totales (ROTACT) como una medida inversa de los costos de agencia. Este ratio mide la eficiencia en la utilización de los activos por parte de los directivos, en donde un bajo valor de este ratio indica toma de decisiones incorrectas, esfuerzos insuficientes, compra de activos improductivos o consumos de perquisites ${ }^{5}$, por lo que existirían altos costos de

${ }^{5}$ Aquellos consumos extraordinarios con carácter discrecional y normalmente no pecuniarios en los que se utilizan recursos superiores a los deseados por los accionistas, como por ejemplo el uso de vehículos de la empresa, oficinas excesivamente lujosas, jets privados, etcétera. 
agencia entre directivos y accionistas (Ang et al., 2000). Por otro lado, un alto valor de este ratio implicaría un mejor aprovechamiento de los activos de la empresa, ya que un mayor incremento de las ventas en relación con los activos supondría un mayor esfuerzo ejecutivo por parte de los directivos.

Entre las variables independientes se abordan la estructura de capital, la concentración de propiedad y la política de dividendos de las firmas, las que serán controladas por la condición del primer accionista en caso de que sea familia, las oportunidades de crecimiento y el tamaño de la empresa. Considerando además el sector industrial y su variación con el tiempo. Dichas variables se describen a continuación.

En primer lugar, la variable a utilizar para medir la deuda será el ratio de endeudamiento (DTAT), el que consiste en la deuda total sobre los activos totales. Adicionalmente, también se considerará el plazo de la deuda como determinante de los costos de agencia, para ello utilizaremos la variable de deuda a corto plazo sobre deuda total (DCDT). Esto, ya que la deuda de corto plazo podría ser más útil que la deuda de largo plazo en la reducción de problemas (Florackis, 2008).

Por otra parte, la concentración de propiedad es medida mediante la proporción de acciones poseídas por el principal accionista (P1). Adicionalmente se incorpora el término de concentración de propiedad al cuadrado (P1CUAD) por la posibilidad de encontrar una relación no lineal con los costos de agencia.

La distribución de dividendos por su parte se medirá con base en la razón entre los dividendos pagados dividido por el patrimonio total (PAYOUT). Es importante mencionar que para esta variable se utiliza el patrimonio en lugar de las utilidades o ingreso neto de la empresa con el propósito de evitar ratios negativos.

En cuanto a las variables de control, se emplea una variable dicotómica, la que indicará si el controlador corresponde a una empresa familiar o no. Así, se estabece la variable (FAM), la que tomará el valor de 1 si la empresa es de naturaleza familiar y 0 si no. 
En este caso cuando los accionistas mayoritarios corresponden a familias, en donde al tener mayores incentivos de monitoreo y control, debiera existir una disminución de los costos de agencia. Sin embargo, dichos accionistas mayoritarios podrían seguir objetivos individuales o familiares alejándose de la maximización de utilidades en desmedro de los accionistas minoritarios. Incluso accionistas mayoritarios podrían contratar a miembros incompetentes de la familia para los puestos ejecutivos de la empresa en lugar de contratar a gerentes competentes (PérezGonzález, 2006).

La magnitud de los costos de agencia relacionados a la subinversión, sustitución de activos y flujo de caja libre, se espera que varíe notoriamente de acuerdo con que si la firma posee altas o bajas oportunidades de crecimiento (Florackis, 2008). Por lo que para medir las oportunidades de crecimiento se utilizará una aproximaxión de la Q de Tobin (QTOBIN), la que consiste en la suma de la capitalización bursátil y la deuda total dividido por los activos totales de la firma.

Del mismo modo, como variable de control también se considera el tamaño de la firma (TAM), la que es establecida como el logaritmo natural de las ventas totales. Es admisible señalar que empresas más grandes pueden disminuir sus costos de agencia a base de economías de escala o alcance, ya que hay un uso más eficiente de los activos (Ang et al., 2000), no obstante, empresas de mayor tamaño suelen presentar mayor separación de la propiedad y control, conduciendo a un aumento de problemas de agencia. Por este motivo, sería interesante ver qué ocurre para el caso chileno.

Finalmente, se señala que la medida de costos de agencia utilizada puede ser afectada por el sector industrial al que pertenecen las empresas. Esto, porque según al sector en donde opera la firma, variará su estructura de costos y el nivel de activos utilizados. Por ello en este trabajo se incluyen ocho variables dummy que representan la clasificación sectorial de las empresas estudiadas 
(DUMMY SECTOR $)^{6}$, así como también variables dummy de tiempo (DUMMY AÑO).

\section{B. Metodología}

El estudio de las variables a contrastar con las hipótesis planteadas anteriormente se llevará a cabo en dos fases. En la primera se realizará un análisis descriptivo en donde se estudiará en términos generales a la empresa chilena basado en estadísticos descriptivos y al análisis de medias. En la segunda etapa se desarrolla un análisis multivariado a base del desarrollo de regresiones utilizando la metodología de datos de panel.

La composición de la muestra permite la combinación de cortes transversales y series de tiempo, lo que permite controlar tanto la heterogeneidad inobservable presente en los datos, así como también los usuales problemas de simultaneidad en los estudios acerca de decisiones empresariales (Arellano y Bover, 1990).

La especificación general de un modelo de regresión con datos de panel es la siguiente:

$$
Y_{i t}=\alpha_{i t}+X \beta_{i t}+\mu_{i t} \quad \operatorname{con} i=1, \ldots \ldots, \mathrm{N} ; \quad \text { y } t=1, \ldots \ldots, \mathrm{T}
$$

Donde $i$ corresponde al individuo o empresa en este caso, $t$ es la dimensión en el tiempo, $\alpha$ es un vector de interceptos de $n$ parámetros, $\beta$ es un vector de $K$ parámetros y $X_{i t}$ es la i-ésima observación al momento $t$ para las $K$ variables explicativas.

Sobre la base de este modelo general se estimaron modelos de datos de panel con modelo MCO agrupado, efecto fijo y efecto aleatorio. Sin embargo, el primero asume que los coeficientes son

${ }^{6}$ Efecto de la industria es medido basado en el código SIC de dos dígitos en el que se establecen ocho sectores industriales para el sistema financiero chileno. 
constantes para todos los individuos en el tiempo, lo que es difícil de asumir considerando la heterogeneidad de las empresas que componen la muestra. Por tanto, solo se analizan los modelos de efectos fijos y efectos variables, para ello se emplea el test de Hausman con el propósito de definir cuál de los dos es el más adecuado en términos econométricos.

De esta forma, los modelos que relacionan los costos de agencia con los mecanismos de control y monitoreo corporativo se definen de la siguiente manera:

Modelo 1:

$$
\begin{aligned}
\text { ROTACT }_{i t}= & \alpha+\beta_{1} \text { DTAT }_{i t}+\beta_{2} \text { FAM }_{i t}+\beta_{3} \text { QTOBIN }_{i t}+\beta_{4} \text { SIZE }_{i t} \\
& +\beta_{5} \text { DUMMYAÑ }_{i t}+\beta_{6} \text { DUMMYSEC }_{i t}+\varepsilon_{i t}
\end{aligned}
$$

Modelo 2:

$$
\begin{aligned}
\text { ROTACT }_{i t}= & \alpha+\beta_{1} D_{C D T_{i t}}+\beta_{2} \text { FAM }_{i t}+\beta_{3} \text { QTOBIN }_{i t}+\beta_{4} \text { SIZE }_{i t} \\
& +\beta_{5} \text { DUMMYAÑ }_{i t}+\beta_{6} \text { DUMMYSEC }_{i t}+\varepsilon_{i t}
\end{aligned}
$$

Modelo 3:

$$
\begin{aligned}
\text { ROTACT }_{i t}= & \alpha+\beta_{1} P_{i t}+\beta_{2} \text { FAM }_{i t}+\beta_{3} \text { QTOBIN }_{i t}+\beta_{4} \text { TAM }_{i t} \\
& +\beta_{5} \text { DUMMYANO }_{i t}+\beta_{6} \text { DUMMYSEC }_{i t}+\varepsilon_{i t}
\end{aligned}
$$

Modelo 4:

$$
\begin{aligned}
\operatorname{ROTACT}_{i t}= & \alpha+\beta_{1} \text { PAYOUT }_{i t}+\beta_{2} \text { FAM }_{i t}+\beta_{3} \text { QTOBIN }_{i t}+\beta_{4} \text { TAM }_{i t} \\
& +\beta_{5} \text { DUMMYAÑO }_{i t}+\beta_{6} \text { DUMMYSEC }_{i t}+\varepsilon_{i t}
\end{aligned}
$$

Modelo 5:

$$
\begin{aligned}
\text { ROTACT }_{i t}= & \alpha+\beta_{1} \text { DTAT }_{i t}+\beta_{2} D C D T_{i t}+\beta_{3} P 1_{i t}+\beta_{4} P_{A Y O U T} \\
& +\beta_{5} \text { FAM }_{i t}+\beta_{6} \text { OTOBIN }_{i t}+\beta_{7} \text { TAM }_{i t} \\
& +\beta_{8} D U M M Y A \tilde{N O}_{i t}+\beta_{9} D \text { UMMYSEC }_{i t}+\varepsilon_{i t}
\end{aligned}
$$


Modelo 6:

$$
\begin{aligned}
\text { ROTACT }_{i t}= & \alpha+\beta_{1} \text { DTAT }_{i t}+\beta_{2} \text { DCDT }_{i t}+\beta_{3} P 1_{i t}+\beta_{4} P 1 C U A D_{i t} \\
& +\beta_{5} \text { PAYOUT }_{i t}+\beta_{6} \text { FAM }_{i t}+\beta_{7} \text { QTOBIN }_{i t} \\
& +\beta_{8} \text { TAM }_{i t}+\beta_{9} D U M M Y A N O_{i t}+\beta_{10} D_{U M M Y S E C}+\varepsilon_{i t}
\end{aligned}
$$

Modelo 7:

$$
\begin{aligned}
\text { ROTACT }_{i t}= & \alpha+\beta_{1} \text { DTAT }_{i t}+\beta_{2} D C D T_{i t}+\beta_{3} P 1_{i t}+\beta_{4} P 1 C U A D_{i t} \\
& +\beta_{5} P \text { AYUT } \\
& +\beta_{6} F_{i t}+\beta_{7} \text { TAM }_{i t} \\
& +\beta_{8} \text { DUMMYANO }_{i t}+\beta_{9} \text { DUMMYSEC }_{i t}+\varepsilon_{i t}
\end{aligned}
$$

En donde el subíndice $i$ indica las empresas que van desde 1 a 113, $t$ son los años que van desde 2003 a 2013 y $\varepsilon_{i t}$ corresponde al término de error que incluye el efecto individual, el temporal y error estocástico.

\section{Resultados}

\section{A. Análisis descriptivo}

En esta sección se describen algunas características de las empresas chilenas a base de los estadísticos descriptivos de las variables utilizadas en este trabajo, y además se realizará un test de diferencia de medias, de tal manera de obtener un diagnóstico más detallado de la situación existente en el mercado chileno. En la tabla 2 podemos ver que en promedio las empresas chilenas no financieras presentan un ratio de ventas totales sobre activos totales de $72 \%$. En relación con el endeudamiento podemos observar que en término medio las empresas chilenas presentan un ratio de deuda total sobre activos totales de $23 \%$, y además se observa que predominan las deudas con vencimiento a largo plazo, ya que el ratio de deuda de corto plazo sobre la deuda total solo alcanza $33 \%$. 
Como ya se ha mencionado anteriormente, Chile se encuentra enmarcado en el modelo continental, lo que tiene sentido al observar $45 \%$ de propiedad en promedio para los primeros accionistas. También se observa que las empresas familiares son predominantes en el mercado chileno alcanzando $69 \%$ en promemdio. Se observa también que en promedio las empresas chilenas pagan $7,6 \%$ de su patrimonio en dividendos.

\section{Tabla 2}

Estadísticos descriptivos de la muestra

\begin{tabular}{|l|c|c|c|c|c|}
\hline VARIABLE & OBS. & MEDIA & DESV. EST. & MÍNIMO & MÁXIMO \\
\hline ROTACT & 1.118 & 0,716 & 0,565 & 0,000 & 4,608 \\
\hline DTAT & 1.118 & 0,232 & 0,144 & 0,000 & 0,731 \\
\hline DCDT & 1.118 & 0,327 & 0,302 & 0,000 & 1,000 \\
\hline P1 & 1.118 & 0,448 & 0,223 & 0,072 & 1,000 \\
\hline PAYOUT & 1.118 & 0,076 & 0,144 & 0,000 & 1,455 \\
\hline FAM & 1.118 & 0,587 & 0,493 & 0,000 & 1,000 \\
\hline QTOBIN & 1.118 & 1,139 & 0,777 & 0,145 & 7,311 \\
\hline TAM & 1.118 & 18,49 & 2,133 & 9,967 & 23,22 \\
\hline
\end{tabular}

En cuanto a la variable Q de Tobin podemos ver que efectivamente existen oportunidades de crecimiento, alcanzando $114 \%(1,14)$, no obstante hay que tener en cuenta la elevada desviación estándar de este resultado y su rango, para un correcto análisis.

Además, en la tabla 3 podemos observar que las variables explicativas utilizadas en los modelos presentan bajo nivel de correlación, exceptuando la relación entre DTAT con SIZE y PAYOUT con QTOBIN, los que mantienen una correlación positiva de 0,41 y 0,5 , respectivamente. De esta forma podemos ver que no 
se presentan correlaciones muy altas que conduzcan a problemas de multicolinealidad en los modelos.

\section{Tabla 3}

Matriz de correlaciones de la muestra

\begin{tabular}{|l|r|r|r|r|r|r|r|r|}
\hline & ROTACT & DTAT & DCDT & P1 & PAYOUT & FAM & QTOBIN & TAM \\
\hline ROTACT & 1,000 & & & & & & & \\
\hline DTAT & 0,092 & 1,000 & & & & & & \\
\hline DCDT & 0,063 & $-0,248$ & 1,000 & & & & & \\
\hline P1 & 0,082 & $-0,081$ & 0,012 & 1,000 & & & & \\
\hline PAYOUT & 0,034 & $-0,134$ & 0,076 & 0,091 & 1,000 & & & \\
\hline FAM & 0,143 & $-0,094$ & 0,034 & $-0,150$ & $-0,152$ & 1,000 & & \\
\hline QTOBIN & $-0,036$ & $-0,065$ & $-0,050$ & 0,037 & 0,499 & $-0,114$ & 1,000 & \\
\hline TAM & 0,379 & 0,411 & $-0,214$ & 0,014 & 0,038 & 0,052 & 0,026 & 1,000 \\
\hline
\end{tabular}

Finalmente se desarrolla un análisis de medias en donde la muestra se dividió en dos partes en función de las variables ROTACT. De esta forma cada variable dependiente estará conformada por dos submuestras separadas por sus respectivas medianas. Entonces, se calcula la media de cada submuestra respecto de cada variable explicativa a analizar, para luego comparar dichas medias con el propósito de determinar la significancia estadística de las diferencias. En la tabla 4 se describen los resultados obtenidos.

Basándonos en los resultados de la tabla 4 podemos ver que las empresas con menores costos de agencia, es decir, con valores de ROTACT sobre la mediana, presentan un mayor nivel de endeudamiento, su deuda es de corto plazo, tienen una concentración de propiedad más alta, mantienen un mayor pago de dividendos, son empresas familiares y presentan altas oportunidades 
de crecimiento. Además se observa que las empresas de menor tamaño tienen menos costos de agencia que las más grandes.

A pesar de que los resultados carecen de precisión necesaria, ya que no constituye un análisis multivariable, en general se apegan a la teoría, dando luces de que mecanismos de control corporativo como el endeudamiento, la concentración de propiedad y el pago de dividendos ayudan a mitigar costos de agencia.

Tabla 4

Análisis de diferencias de medias

\begin{tabular}{|l|c|c|c|}
\hline & \multicolumn{3}{|c|}{ VARIABLE DEPENDIENTE ROTACT } \\
\hline VARIABLES & $\begin{array}{c}\text { MEDIA DE ROTACT } \\
\text { BAJO LA MEDIANA }\end{array}$ & $\begin{array}{c}\text { MEDIA DE ROTACT } \\
\text { SOBRE LA MEDIANA }\end{array}$ & $\begin{array}{c}\text { DIF. MEDIAS } \\
\text { (SIGNIFIC.) }\end{array}$ \\
\hline DTAT & 0,223 & 0,240 & $-0,018$ \\
\hline & & & 0,042 \\
\hline DCDT & 0,283 & 0,371 & $-0,087$ \\
\hline & & & 0,000 \\
\hline P1 & 0,429 & 0,467 & $-0,037$ \\
\hline & & & 0,005 \\
\hline PAYOUT & 0,051 & 0,102 & $-0,051$ \\
\hline & & & 0,000 \\
\hline FAM & 0,549 & 0,624 & $-0,075$ \\
\hline & & & 0,011 \\
\hline QTOBIN & 1,036 & 1,241 & $-0,206$ \\
\hline & & & 0,000 \\
\hline SIZE & 17,79 & 19,17 & $-1,378$ \\
\hline & & & 0,000 \\
\hline
\end{tabular}

B. Análisis multivariado

En esta sección se analizan los resultados obtenidos empíricamente y se contrastan con las hipótesis establecidas para este trabajo. En primer lugar se analizan los modelos 1 a 5 para estudiar tanto los 
efectos individuales como en su forma conjunta de las variables explicativas sobre los costos de agencia y posteriormente se analiza el modelo 6, en el que se agrega la variable P1CUAD con el propósito de observar si la concentración de propiedad presenta un comportamiento no lineal. Finalmente, a base del modelo 6, la muestra se divide en dos partes iguales correspondientes a oportunidades de crecimiento altas y bajas, de tal manera de analizar el impacto de los mecanismos de control según el nivel de oportunidades de crecimiento que presentan las empresas. Es importante señalar que para cada estimación de los modelos se utilizaron errores robustos con el propósito de aminorar potenciales problemas de heterocedasticidad.

Los resultados obtenidos en los modelos 1 a 5 se exponen en la tabla 5. Para cada modelo se efectuó el test de Hausman, el que es presentado en la tabla como forma de medir la pertinencia del procedimiento de estimación elegido.

Como podemos ver en la tabla 6, tanto en el modelo 1 como en el 5 se puede apreciar que el ratio de endeudamiento DTAT presenta una relación negativa y significativa al 1\% con ROTACT. Esto indica que mientras mayor sea la eficiencia en la utilización de activos (menores costos de agencias) las empresas se endeudarán menos, tomando un comportamiento opuesto al planteado en la hipótesis 1. Obteniendo similares resultados a los encontrados por Singh y Davidson (2003) para empresas grandes en el mercado estadounidense.

En cuanto a la deuda a corto plazo en el modelo 2 y 5 se observa que el coeficiente es positivo y estadísticamente significativo a un nivel de confianza del $95 \%$. Este resultado va acorde a lo establecido en la hipótesis 2 , ya que el endeudamiento a corto plazo funciona como mecanismo para disminuir los costos de agencia. 


\section{Tabla 5}

\section{Coeficientes estimados y errores estándar (variable dependiente ROTACT)}

\begin{tabular}{|c|c|c|c|c|c|}
\hline VARIABLES & MODELO 1 & MODELO 2 & MODELO 3 & MODELO 4 & MODELO 5 \\
\hline \multirow[t]{2}{*}{ DTAT } & $-0,592 * * *$ & & & & $-0,559 * * *$ \\
\hline & $-0,143$ & & & & $-0,144$ \\
\hline \multirow[t]{2}{*}{ DCDT } & & $0,097 * *$ & & & $0,079 * *$ \\
\hline & & $-0,039$ & & & $-0,038$ \\
\hline \multirow[t]{2}{*}{ P1 } & & & 0,048 & & 0,023 \\
\hline & & & $-0,146$ & & $-0,138$ \\
\hline \multirow[t]{2}{*}{ PAYOUT } & & & & $0,213^{* *}$ & $0,177^{* *}$ \\
\hline & & & & $-0,084$ & $-0,073$ \\
\hline \multirow[t]{2}{*}{ FAM } & 0,093 & 0,115 & 0,119 & 0,122 & 0,099 \\
\hline & $-0,091$ & $-0,09$ & $-0,091$ & $-0,091$ & $-0,091$ \\
\hline \multirow[t]{2}{*}{ QTOBIN } & $0,062 * * *$ & $0,061 * * *$ & $0,059 * * *$ & $0,046 * * *$ & $0,053 * * *$ \\
\hline & $-0,016$ & $-0,015$ & $-0,016$ & $-0,017$ & $-0,015$ \\
\hline \multirow[t]{2}{*}{ TAM } & $0,176^{* * *}$ & $0,163 * * *$ & $0,162 * * *$ & $0,161 * * *$ & $0,175^{* * *}$ \\
\hline & $-0,018$ & $-0,018$ & $-0,018$ & $-0,018$ & $-0,018$ \\
\hline \multirow[t]{2}{*}{ CONSTANTE } & $-2,409 * * *$ & $-2,386^{* * *}$ & $-2,357 * * *$ & $-2,309 * * *$ & $-2,447 * * *$ \\
\hline & $-0,341$ & $-0,345$ & $-0,349$ & $-0,353$ & $-0,335$ \\
\hline DUMMY SECTOR & Sí & Sí & Sí & Sí & Sí \\
\hline DUMMY AÑO & Sí & Sí & Sí & Sí & Sí \\
\hline OBSERVACIONES & 1.118 & 1.118 & 1.118 & 1.118 & 1.118 \\
\hline R CUADRADO & 0,462 & 0,435 & 0,429 & 0,436 & 0,469 \\
\hline TEST F & $216,5^{* * *}$ & $183,9 * * *$ & $156,9 * * *$ & $165,7 * * *$ & $242,9 * * *$ \\
\hline TEST HAUSMAN & 17,72 & 23,44 & 12,38 & 12,48 & 22,22 \\
\hline NÚMERO EMPRESAS & 113 & 113 & 113 & 113 & 113 \\
\hline
\end{tabular}

Notas: Errores estándar entre paréntesis.

$*, * *$ y $* * *$ representan el nivel de significancia a un $10 \%, 5 \%$ y $1 \%$ respectivamente.

La variable dependiente es ROTACT. Basado en el test de Hausman, para todos los modelos se utilizó efectos aleatorios.

Al analizar la concentración de propiedad de las empresas, se puede apreciar que la variable referente al porcentaje de propiedad poseída por el primer accionista P1 tiene una relación positiva con ROTACT, sin embargo esta no es significativa, indicando de esta forma un escaso impacto sobre la medida de costos de agencia. 
En cuanto al pago de dividendos (PAYOUT), se observa que tanto el modelo 4 como el 5 presentan una relación positiva y significativa al $1 \%$ con ROTACT. Lo que es consistente con la teoría, ya que un mayor pago de dividendos implicará una disminución de costos de agencia. Es factible mencionar que las empresas chilenas se rigen por la Ley $\mathrm{N}^{\circ} 18.046$, la que señala que las sociedades anónimas chilenas tienen la obligación de pagar dividendos de al menos el 30\% de sus utilidades líquidas de cada ejercicio. Por este motivo dicha ley es un factor clave en el impacto que tiene la política de dividendos de las empresas chilenas sobre los costos de agencia, lo que es consistente con los resultados obtenidos.

La variable FAM correspondiente a empresas en donde el primer accionista es una familia, arroja un coeficiente positivo pero estadísticamente no significativo, por lo que no presenta mayor relevancia en el modelo. Por otro lado, las oportunidades de crecimiento medidas por la Q de Tobin (QTOBIN) presentan una relación positiva y significativa con ROTACT para los cinco modelos, sugiriendo que empresas con altas oportunidades de crecimiento son más efectivas en la reducción y control de costos de agencia. Esto indica que empresas con bajas oportunidades de crecimiento presentan mayores costos de agencia, lo que sugiere problemas de agencia asociados con el flujo de caja de las firmas (Jensen, 1986).

Por último, al observar la variable correspondiente al tamaño de la empresa (TAM) los resultados arrojan coeficientes positivos y significativos al 1\%, señalando que mientras mayor sea el tamaño de la empresa, la eficiencia en la utilización de activos (ROTACT) será mayor, por lo que existirán menores problemas de agencia. Esto puede explicarse en parte por el hecho de que las grandes empresas tienen acceso a una gama más amplia de recursos para la mitigación o control de los conflictos de agencia entre accionistas y directivos.

En términos generales, se puede ver que todos los modelos muestran un alto grado de significancia global alcanzando un nivel 
de confianza superior al $99 \%$, es decir, los parámetros como un todo son significativos. Además, los coeficientes de determinación (RCUADRADO) son bastante aceptables superando el $40 \%$ de explicabilidad por parte de las variables independientes.

En la tabla 6 se expone el modelo 6 en el que se incorpora la variable P1CUAD utilizado para corroborar la existencia de un comportamiento no lineal en el efecto de la concentración de propiedad sobre los costos de agencia. Además, se muestran los resultados al dividir la muestra en altas y bajas oportunidades de crecimiento, porque es probable que la magnitud de los costos de agencia difiera según el nivel de oportunidades de crecimiento que presente la firma.

Al observar los resultados arrojados por el modelo 6, en general se repite la dirección con que cada mecanismo de control afecta en los costos de agencia. Sin embargo, al incluir la variable P1CUAD se obtiene un resultado bastante interesante en relación con la concentración de propiedad. La variable P1, que mide el porcentaje de participación accionaria del primer accionista, arroja un coeficiente estadísticamente significativo relacionándose positiva-mente con ROTACT, indicando que una empresa más concentrada será más eficiente en la utilización de los activos. No obstante la variable P1 al cuadrado (P1CUAD), deja en evidencia una relación negativa y significativa al $1 \%$, lo que sugiere que a medida que aumenta la concentración de la propiedad, es más probable que al llegar a un cierto punto se traduzca en costos de agencia más altos, lo que refleja el poder de los grandes accionistas de expropiar recursos de la empresa y tomar decisiones para extraer beneficios privados. 
Tabla 6

Coeficientes estimados y errores estándar incluyendo P1CUAD y controlando por oportunidades de crecimiento

\begin{tabular}{|c|c|c|c|}
\hline \multirow[b]{2}{*}{ VARIABLES } & \multirow[b]{2}{*}{ MODELO 6} & \multicolumn{2}{|c|}{ MODELO 7} \\
\hline & & $\begin{array}{l}\text { ALTAS OPORTUNIDADES } \\
\text { DE CRECIMIENTO }\end{array}$ & $\begin{array}{c}\text { BAJAS } \\
\text { OPORTUNIDADES } \\
\text { DE CRECIMIENTO }\end{array}$ \\
\hline \multirow[t]{2}{*}{ DTAT } & - & $-0,538 * * *$ & $-0,737 * * *$ \\
\hline & $-0,14$ & $-0,16$ & $-0,27$ \\
\hline \multirow[t]{2}{*}{ DCDT } & $0,075 * *$ & 0,067 & 0,06 \\
\hline & $-0,035$ & $-0,047$ & $-0,057$ \\
\hline \multirow[t]{2}{*}{ P1 } & & $1,338^{*}$ & $1,727 * * *$ \\
\hline & $-0,625$ & $-0,719$ & $-0,582$ \\
\hline \multirow[t]{2}{*}{ P1CUAD } & - & $-1,171 *$ & $-1,749 * * *$ \\
\hline & $-0,584$ & $-0,643$ & $-0,534$ \\
\hline \multirow[t]{2}{*}{ PAYOUT } & $0,172 * *$ & $0,324 * * *$ & 0,135 \\
\hline & $-0,069$ & $-0,089$ & $-0,14$ \\
\hline \multirow[t]{2}{*}{ FAM } & 0,101 & 0,127 & 0,073 \\
\hline & $-0,091$ & $-0,089$ & $-0,112$ \\
\hline \multirow[t]{2}{*}{ QTOBIN } & $0,055^{* * * *}$ & & \\
\hline & $-0,015$ & & \\
\hline \multirow[t]{2}{*}{ TAM } & & $0,116^{* * *}$ & $0,190 * * *$ \\
\hline & $-0,017$ & $-0,019$ & $-0,022$ \\
\hline \multirow[t]{2}{*}{ CONSTANTE } & - & $-1,683 * * *$ & $-2,952 * * *$ \\
\hline & $-0,358$ & $-0,436$ & $-0,422$ \\
\hline DUMMY SECTOR & Sí & Sí & Sí \\
\hline DUMMY AÑO & Sí & Sí & Sí \\
\hline OBSERVACIONES & 1.118 & 559 & 559 \\
\hline R CUADRADO & 0,493 & 0,324 & 0,509 \\
\hline TEST F & $253,4 * * *$ & $133,5 * * *$ & $198,9 * * *$ \\
\hline TEST HAUSMAN & 25,49 & $27,45^{*}$ & $16,47^{*}$ \\
\hline NÚMERO & 113 & 95 & 93 \\
\hline
\end{tabular}

Notas: Errores estándar entre paréntesis.

$*$, ** y *** representan el nivel de significancia a un $10 \%, 5 \%$ y $1 \%$ respectivamente.

La variable dependiente es ROTACT. Basado en el test de Hausman, para todos los modelos se utilizó efectos aleatorios. 
Finalmente, para medir el impacto de la estructura de capital, la concentración de propiedad y la política de dividendos en los costos de agencia a base de empresas con altas o bajas oportunidades de crecimiento, la muestra se separa en dos partes iguales respecto de la mediana de la variable Q de Tobin.

Los resultados del modelo 7 muestran que el nivel de endeudamiento presenta una relación negativa con la eficiencia en la utilización de activos, por lo que empresas más endeudadas tendrán mayores costos de agencia con independencia del nivel de oportunidades de crecimiento. Otro resultado relevante tiene relación con la concentración de propiedad, en donde se observa un efecto no lineal sobre los costos de agencia de una manera mucho más significativa en un contexto de bajas oportunidades de crecimiento. En ese sentido, en el caso que la empresa disponga de bajas oportunidades de crecimiento, y los directivos tengan incentivos a invertir en proyectos de VAN negativo, el control de los accionistas mayoritarios puede manejar el comportamiento discrecional. Sin embargo, una excesiva concentración lleva a la posibilidad de expropiación de riquezas de los accionistas minoritarios.

Los resultados también arrojan que para altas oportunidades de inversión existe una relación positiva entre ROTACT y la variable de pago de dividendos a un nivel del 1\%. Este resultado implica que empresas con altas oportunidades de crecimiento son más propensas a pagar mayores niveles de dividendos con el propósito de emitir información favorable sobre expectativas futuras, así como también reducir asimetrías de información entre controladores y accionistas minoritarios.

Por último se aprecia una relación positiva entre el tamaño de la empresa y la eficiencia en la utilización de activos, no obstante, esta relación es independiente del nivel de oportunidades rentables de inversión. 


\section{Conclusiones}

El presente trabajo se enfoca en analizar el impacto de ciertos mecanismos corporativos de control, como la estructura de capital, la concentración de propiedad y la política de dividendos sobre la discrecionalidad directiva en empresas chilenas no financieras.

La discrecionalidad de los directivos, traducida en costos de agencia, se expresó mediante el ratio de rotación de activos. Dicho ratio actúa como una medida inversa de los costos de agencia refiriéndose a la eficiencia directiva en la utilización y aprovechamiento de estos.

Sobre la base de los resultados obtenidos en este trabajo, en primer lugar se puede apreciar que la estructura de capital no se comporta como un mecanismo para mitigar costos de agencia, sino más bien pareciera que el endeudamiento de las empresas favorece la discrecionalidad directiva en el mercado chileno. La explicación a esta situación tiene que ver con las características propias del mercado financiero chileno en donde la principal fuente de financiamiento de las empresas chilenas es la deuda bancaria, por lo que se puede generar un problema de selección adversa, porque empresas con altos niveles de costos de agencias solo tengan la posibilidad de conseguir deuda crediticia ante la incapacidad de encontrar otro tipo de financiamiento en los mercados de capitales. Sin embargo, existe evidencia que cuando la deuda es a corto plazo la divergencia de intereses disminuye entre directivos y accionistas. Esto puede ser explicado por el hecho de que si la deuda tiene vencimiento a corto plazo, los directivos estarán sometidos a un control más frecuente debido a la constante renovación de contratos de deuda, es decir, existirá una frecuente supervisión por parte de las instituciones financieras o acreedores.

En cuanto a la concentración de propiedad, tanto para altas como para bajas oportunidades de crecimiento presentes en la empresa, se observa un efecto no lineal sobre los costos de agencia. La mayor concentración de propiedad accionarial constituirá un 
mecanismo de control para la firma, no obstante, para niveles más elevados pareciera producirse un efecto de expropiación de los accionistas mayoritarios sobre los minoritarios. Lo anterior puede ser causado por las estructuras piramidales y grupos económicos, en donde mientras los accionistas controladores apuntarán a la generación de utilidades de las diferentes sociedades controladas por el grupo económico, los accionistas minoritarios solo buscarán las utilidades de la empresa de la que son accionistas, generando conflictos de intereses entre ambas partes.

En relación con la política de dividendos, se observa que el efecto del pago de dividendos sobre el nivel de eficiencia sobre los activos es positivo y más significativo en presencia de altas oportunidades de crecimiento. Esto se apega a la teoría, ya que los directivos pueden utilizar el pago de dividendos como una señal confiable para transmitir información privada sobre flujos de caja y perspectivas futuras de la empresa, de tal manera de reducir asimetrías de información entre directivos e inversores externos. Además, este resultado tiene sentido al considerar la Ley $\mathrm{N}^{\mathrm{o}} 18.046$, la que indica que las sociedades anónimas chilenas tienen la obligación de pagar al menos el 30\% de sus utilidades líquidas de cada ejercicio, siendo este un mecanismo importante de protección a los accionistas minoritarios y, por tanto, en la reducción de costos de agencia.

A pesar que los resultados han sido en general satisfactorios, este trabajo presenta algunas limitaciones principalmente en relación con la carencia de datos respecto de la participación accionarial de los directivos (managers) y de los directores de las empresas, impidiendo de esta forma un estudio mucho más preciso acerca de la implicancia de los mecanismos de gobierno corporativo en los costos de agencia. Además, sería conveniente profundizar en función a otras medidas de agencia que permitan analizar las distintas situaciones de agencia, como los conflictos entre accionistas y acreedores o accionistas mayoritarios y minoritarios. 


\section{Referencias}

ANG, J.S., R.A. COLE and J.W. LIN (2000), "Agency costs and ownership structure", The Journal of Finance, Vol. 55(1), pp. 81-106.

Arellano, M. and O. Bover (1990), "La econometría de datos de panel", Investigaciones Económicas, Vol. 14(1), pp. 3-45.

KraAkman, R., P. Davies, J. Armour, H.B. Hansmann, G. Hertig, L. ENRIQUES and K.J. HOPT (2009), "The anatomy of corporate law: a comparative and functional approach", Oxford University Press on Demand.

Custódio, C., M.A. Ferreira and L. Laureano (2013), "Why are US firms using more short-term debt?", Journal of Financial Economics, Vol. 108(1), pp. 182-212.

DewENTER, K.L. and V.A. WARTHER (1998), "Dividends, asymmetric information, and agency conflicts: Evidence from a comparison of the dividend policies of Japanese and US firms", The Journal of Finance, Vol. 53(3), pp. 879-904.

DiAMOND, D.W. (1991), "Debt maturity structure and liquidity risk", The Quarterly Journal of Economics, Vol. 106(3), pp. 709-737.

EASTERBROOK, F.H. (1984), "Two agency-cost explanations of dividends", The American Economic Review, Vol. 74(4), pp. 650-659.

FAMA, E.F. and M.C. JENSEN (1983), "Separation of ownership and control", The Journal of Law and Economics, Vol. 26(2), pp. 301-325.

FLORACKIs, C. (2008), "Agency costs and corporate governance mechanisms: Evidence for UK firms", International Journal of Managerial Finance, Vol. 4(1), pp. 37-59.

FRIEND, I. and L.H. LANG (1988), "An empirical test of the impact of managerial self-interest on corporate capital structure", The Journal of Finance, Vol 43(2), pp. 271-281.

GutiÉRrez, M.G. (2008), Costes de agencia y de transacción como determinantes de las decisiones financieras: un análisis de ecuaciones estructurales. (Doctoral dissertation, Universidad Complutense de Madrid). 
JENSEN, M.C. (1986), "Agency costs of free cash flow, corporate finance, and takeovers", The American Economic Review, Vol. 76(2), pp. 323-329.

Jensen, M.C. and W.H. Meckling (1976), "Theory of the firm: Managerial behavior, agency costs and ownership structure", Journal of Financial Eeconomics, Vol. 3(4), pp. 305-360.

LÓPEZ, F. and P. SAONA (2007), "Endeudamiento, dividendos y estructura de propiedad como determinantes de los problemas de agencia en la gran empresa española", Cuadernos de Economía y Dirección de la Empresa, Vol. 10(31), pp. 119-146.

La Porta, R., F. Lopez-DE-Silanes and A. Shleifer (1998), "Law and finance", The Journal of Political Economy”, Vol. 108(6), pp. 1131-1155.

LA Porta, R., F. Lopez-DE-Silanes and A. Shleifer (1999), "Corporate ownership around the world", The Journal of Finance, Vol. 54(2), pp. 471-517.

LEFORT, F. (2003), “Gobierno corporativo:¿qué es? y ¿cómo andamos por casa?”, Cuadernos de Eeconomía, Vol. 40(120), pp. 207-237.

LEFORT, F. and R. GONZÁLEZ (2008), "Hacia un mejor gobierno corporativo en Chile", Revista Abante, Vol. 11(1), pp. 17-37.

MYERS, S.C. (1977), "Determinants of corporate borrowing”, Journal of Financial Economics, Vol. 5(2), pp. 147-175.

PÉREZ-GONZÁleZ, F. (2006), "Inherited control and firm performance", American Economic Review, Vol. 96(5), pp. 1559-1588.

SHLEIFER, A. and R.W. ViShNY (1997), “A survey of corporate governance”, The Journal of Finance, Vol. 52(2), pp. 737-783.

SINGH, M. and W.N. DAVIDSON III (2003), "Agency costs, ownership structure and corporate governance mechanisms", Journal of Banking \& Finance, Vol. 27(5), pp. 793-816.

SMith, ClifFord JR. and Ross L. WATtS (1992), "The investment opportunity set and corporate financing, dividend, and compensation policies", Journal of Financial Economics, Vol. 32(3), pp. 263-292. 
SMITH JR, C.W. and J.B. WARNER, J (1979), "On financial contracting: An analysis of bond covenants", Journal of Fnancial Economics, Vol. 7(2), pp. 117-161.

StULZ, R. (1990), "Managerial discretion and optimal financing policies", Journal of Financial Economics, Vol. 26(1), pp. 3-27. 